\title{
EOPs: ¿La cinugía de bypass coronario ofrece menor morbimortalidad que la intervención coronaria percutánea en pacientes con diabetes tipo 2 y enfermedad coronaria multivaso?
}

Does coronary bypass surgery offers less morbidity and mortality than percutaneous coronary intervention in patients with type 2 diabetes and multivessel coronary artery disease?

Leandro Barbagelata*

\begin{abstract}
Resumen
A propósito de una viñeta clínica de un paciente con diabetes y enfermedad coronaria multivaso se revisa la evidencia sobre cuál es la mejor alternativa terapéutica.
\end{abstract}

\section{Abstract}

After the description of a clinical vignette of a patient with diabetes and multivessel coronary artery disease, it is reviewed the evidence about the best therapeutic alternative.

Barbagelata L. ¿La cirugía de Bypass coronario ofrece menor morbimortalidad que la Intervención coronaria percutánea en pacientes con diabetes tipo 2 y enfermedad coronaria multivaso?. Evid Act Pract Ambul. Ene-Mar 2014;17(1):37-38.

\section{Introducción}

Se presenta al consultorio de medicina familiar un paciente de sexo masculino de 59 años, con antecedentes de diabetes tipo 2 diagnosticada hace aproximadamente 11 años, con hipertensión controlada y dislipidemia. El señor refería angor de esfuerzo de dos meses de evolución. La doctora le solicitó una ergometría que resultó positiva con infradesnivel del segmento ST en la cara anterolateral izquierda. El paciente fue remitido al servicio de cardiología para seguir siendo estudiado. Se le realizó una cinecoronariografía que diagnosticó enfermedad coronaria multivaso, con afección de la arteria descendente anterior, la coronaria derecha y la arteria circunfleja, todas con lesiones significativas. Por tal motivo se le propone realizar una cirugía de revascularización arterial con bypass. Me pregunto en este caso porqué se opta por la técnica de Bypass y no por la intervención coronaria percutánea (angioplastia).

\section{Pregunta que generó el caso}

En pacientes con diabetes tipo 2 (hombres y mujeres mayores de 45 años) con enfermedad coronaria multivaso, ¿existe evidencia, en cuanto morbimortalidad, de que el Bypass coronario es una mejor técnica de revascularización que la intervención coronaria percutánea?

\section{Estrategia de búsqueda}

Se realizó una búsqueda en Pubmed, utilizando las siguientes palabras claves "revascularization AND type 2 diabetic AND coronarydisease", restringiendola a ensayos clínicos y a artículos publicados en los últimos cinco años. Fueron identificadas 32 citas de las cuales tres fueron utilizadas para responder la pregunta.

\section{Enfermedad coronaria multivaso y diabetes tipo 2}

El concepto de enfermedad coronaria multivaso, abarca la disminución del flujo sanguíneo por estenosis luminal de dos o más arterias coronarias o del tronco coronario.

La diabetes es considerada un factor de riesgo cardiovascular debido a que contribuye y acelera la génesis del fenómeno de disfunción endotelial en los vasos arteriales, lo que a su vez contribuye progresivamente a la formación y progresión de la placa ateroesclerótica.

La enfermedad coronaria es la principal causa de muerte en pacientes con diabetes, y además, estas personas presentan una mayor probabilidad de desarrollar dicha patología y de sufrir un evento cardiovascular (IAM, ICC, ACV, angor, entre otros), si se los compara con la población no diabética.

En la actualidad las dos técnicas de revascularización miocárdica que se utilizan son la intervención coronaria percutánea (IPC) y la cirugía de bypass arterial (en inglés: CABG).

\section{Resumen de evidencia}

Bansilal S, Farkouh M, Hueb W, Ogdie M, Dangas G, Lansky A $\mathrm{y}$ col. The Future Revascularization Evaluation in patients with Diabetes mellitus: optimal management of Multivesseldisease (FREEDOM) trial: clinical and angiographic profile at study entry.

Am Heart J. 2012 Oct;164(4):591-9.

doi: 10.1016/j.ahj.2012.06.012.

El estudio incluyó 1.900 participantes con diabetes de todo el mundo con enfermedad coronaria multivaso que fueron aleatorizados a intervención coronaria percutánea (sigla en inglés: IPC) o bypass coronario (sigla en inglés: CABG).

El estudio comenzó en abril de 2005 y finalizó marzo 2010. Durante una mediana de seguimiento de 3,8 años, el resultado primario compuesto ocurrió significativamente menos en el grupo CABG que en el grupo ICP, y también fue menor la incidencia de infarto de miocardio (IAM), y la mortalidad global y cardiovascular a cinco años (tabla 1).

La incidencia de accidente cerebrovascular (ACV) fue significativamente mayor en el grupo CABG, principalmente por el exceso de ACV durante los primeros 30 días. De los accidentes $\mathrm{ACV}$, la mayoría (87\%) fueron isquémicos y $13 \%$, hemorrágicos.

Tabla 1. Resultados primarios de los individuos incluidos en el ensayo FREEDOM a cinco años de la aleatorización.

\begin{tabular}{l|c|c|c|c}
\multicolumn{1}{c|}{ Resultado } & $\begin{array}{c}\text { Cirugia } \\
\text { de by pass } \\
\text { coronário } \\
\mathbf{n = 9 4 7}\end{array}$ & $\begin{array}{c}\text { Intervención } \\
\text { coronaria } \\
\text { percutánea } \\
\mathbf{n = 9 5 3}\end{array}$ & RR (IC95\%) & NNT (IC95\%) \\
\hline Primario combinado combinado & $18,7 \%$ & $26,6 \%$ & $0,70(0,59 \mathrm{a} \mathrm{0,83)}$ & 13 (9 a 24) \\
\hline Todas las causas de muerte & $10,9 \%$ & $16,3 \%$ & $0,67(0,53 \mathrm{a} 0,84)$ & 19 (12 a 43) \\
\hline Infarto agudo de miocardio & $6,0 \%$ & $13,9 \%$ & $0,43(0,32 \mathrm{a} 0,59)$ & 13 (10 a 19) \\
\hline Accidente cerebrovascular & $5,2 \%$ & $2,4 \%$ & $2,14(1,32 \mathrm{a} 3,49$ & 36 (22 a 100) \\
\hline Muerte cardiovascular & $6,8 \%$ & $10,9 \%$ & 0,62 (0,46 a 0,83) & 24 (15 a 63) \\
\hline
\end{tabular}

Resultado primario combinado: todas las causas de muerte y además, IAM y ACV no fatales.

* Alumno del Instituto Universitario Hospital Italiano de Buenos Aires. leandro.barbagelata @ hiba.org.ar 
Hlatky M, Boothroyd B, Bravata D y col. Coronary artery bypass surgery compared with percutaneous coronary interventions for multivessel disease: a collaborative analysis of individual patient data from ten randomised trials. Lancet 2009;373:1190-1197.

Se trata de una revisión sistemática de diez ensayos aleatorizados que habían incluido 7812 pacientes con enfermedad coronaria multivaso (ECMV) con el objetivo de comparar la eficacia de CABG con la de IPC (media de seguimientos 5,6 años). En la rama de pacientes con ECMV sin diabetes, en términos de morbimortalidad no hubo diferencias en los resultados comparando ambas técnicas. Sin embargo en la rama de pacientes con ECMV y diabetes, la mortalidad en el grupo tratado con CABG $(23 \%)$ fue menor que la del grupo asignado a IPC (29\%). Ver figura 1.

Figura 1. Mortalidad en pacientes con diabetes y sin diabetes asignados a CABG o PCl (10 ensayos).

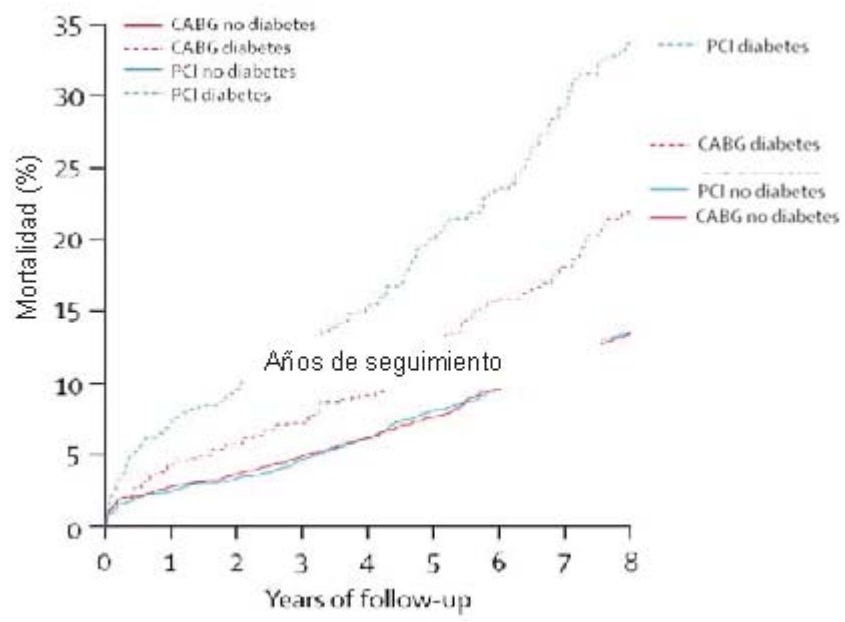

\section{Conclusión}

Existe fuerte evidencia científica a favor de que la técnica de revascularización miocárdica se asocia a menor morbimortalidad a largo plazo en pacientes con diabetes tipo 2 con enfermedad coronaria multivaso es la CABG.

Sin embargo esta técnica se asoció a una mayor incidencia de ACV en comparación con la IPC. Se recomienda que estos pacientes sean evaluados por un equipo interdisciplinario (cardiólogos, cirujanos cardiovasculares, anestesiólogos) para que el paciente pueda tomar una decisión contando con la mejor evidencia disponible. 